\title{
Rh-CXCL-12 Attenuates Neuronal Pyroptosis after Subarachnoid Hemorrhage in Rats via Regulating the CXCR4/NLRP1 Pathway
}

\author{
Ran Gu, ${ }^{1}$ Lu Wang, ${ }^{1}$ Hao Zhou, ${ }^{2}$ Xike Wang, ${ }^{2}$ Cameron Lenahan, ${ }^{3}$ Hao Qu, ${ }^{1}$ Yonghe Liu, ${ }^{4}$ \\ Shirong Li, ${ }^{1}$ Changxiu Wei, ${ }^{1}$ Lu Han, ${ }^{1}$ Xiao Hu $(1),{ }^{1}$ and Gang Zuo ${ }^{5}$ \\ ${ }^{1}$ Department of Neurology, Guizhou Provincial People's Hospital, Guiyang 550002, China \\ ${ }^{2}$ Department of Pediatric, Guizhou Provincial People's Hospital, Guiyang 550002, China \\ ${ }^{3}$ Burrell College of Osteopathic Medicine, Las Cruces, NM 88003, USA \\ ${ }^{4}$ Department of Clinical Laboratory, Guizhou Provincial People's Hospital, Guiyang 550002, China \\ ${ }^{5}$ Department of Neurosurgery, Taicang Hospital Affiliated to Soochow University, Taicang, Suzhou, Jiangsu 215400, China
}

Correspondence should be addressed to Xiao Hu; 18096105119@163.com and Gang Zuo; zg13771721907@163.com

Received 22 August 2021; Accepted 19 October 2021; Published 9 November 2021

Academic Editor: Aldrin Gomes

Copyright (C) 2021 Ran Gu et al. This is an open access article distributed under the Creative Commons Attribution License, which permits unrestricted use, distribution, and reproduction in any medium, provided the original work is properly cited.

\begin{abstract}
Subarachnoid hemorrhage $(\mathrm{SAH})$ is a cerebrovascular disease associated with high morbidity and mortality. CXCR4 provides neuroprotective effects, which can alleviate brain injury and inflammation induced by stroke. Previous studies have suggested that CXCR4 reduces the pyroptosis of LPS-stimulated BV2 cells. The purpose of this study was to evaluate the antipyroptosis effects and mechanisms of CXCR4 after SAH. SAH animal model was induced via endovascular perforation. A total of 136 male SpragueDawley rats were used. Recombinant human cysteine-X-cysteine chemokine ligand 12 (rh-CXCL-12) was administered intranasally at $1 \mathrm{~h}$ after SAH induction. To investigate the underlying mechanism, the inhibitor of CXCR4, AMD3100, was administered intraperitoneally at $1 \mathrm{~h}$ before SAH. The neurobehavior tests were assessed, followed by performing Western blot and immunofluorescence staining. The Western blot results suggested that the expressions of endogenous CXCL-12, CXCR4, and NLRP1 were increased and peaked at $24 \mathrm{~h}$ following SAH. Immunofluorescence staining showed that CXCR4 was expressed on neurons, microglia, and astrocytes. Rh-CXCL-12 treatment improved the neurological deficits and reduced the number of FJCpositive cells, IL-18-positive neurons, and cleaved caspase-1(CC-1)-positive neurons after SAH. Meanwhile, rh-CXCL-12 treatment increased the levels of CXCL-12 and CXCR4, and reduced the levels of NLRP1, IL-18, IL-1 $\beta$, and CC-1. Moreover, the administration of AMD3100 abolished antipyroptosis effects of CXCL-12 and its regulation of CXCR4 post-SAH. The CXCR4/NLRP1 signaling pathway may be involved in CXCL-12-mediated neuronal pyroptosis after SAH. Early administration of CXCL-12 may be a preventive and therapeutic strategy against brain injury after SAH.
\end{abstract}

\section{Background}

The novel coronavirus disease 2019 (COVID-19) is a global pandemic with international concern. Aneurysmal subarachnoid hemorrhage ( $\mathrm{SAH}$ ) is a devastating and life-threatening disease associated with high mortality and disability $[1,2]$. The annual worldwide incidence of SAH is approximately 9.1 per 100,000 people [3], and SAH resulting from intracranial aneurysm rupture accounts for $5-10 \%$ of strokes [4]. There is an association between the SAH and COVID-19 infection [5]. Early brain injury (EBI) appears in the first 3 days following $\mathrm{SAH}$, which is the major cause of poor prognosis regarding high mortality and delayed neurological deficits.

EBI begins immediately after a ruptured intracranial aneurysm, which has been proven to be the primary cause of poor outcome after SAH. During the EBI period, ruptured intracranial aneurysms result in many physiological derangements, including elevated intracranial pressure, decreased cerebral blood flow, and global cerebral ischemia 


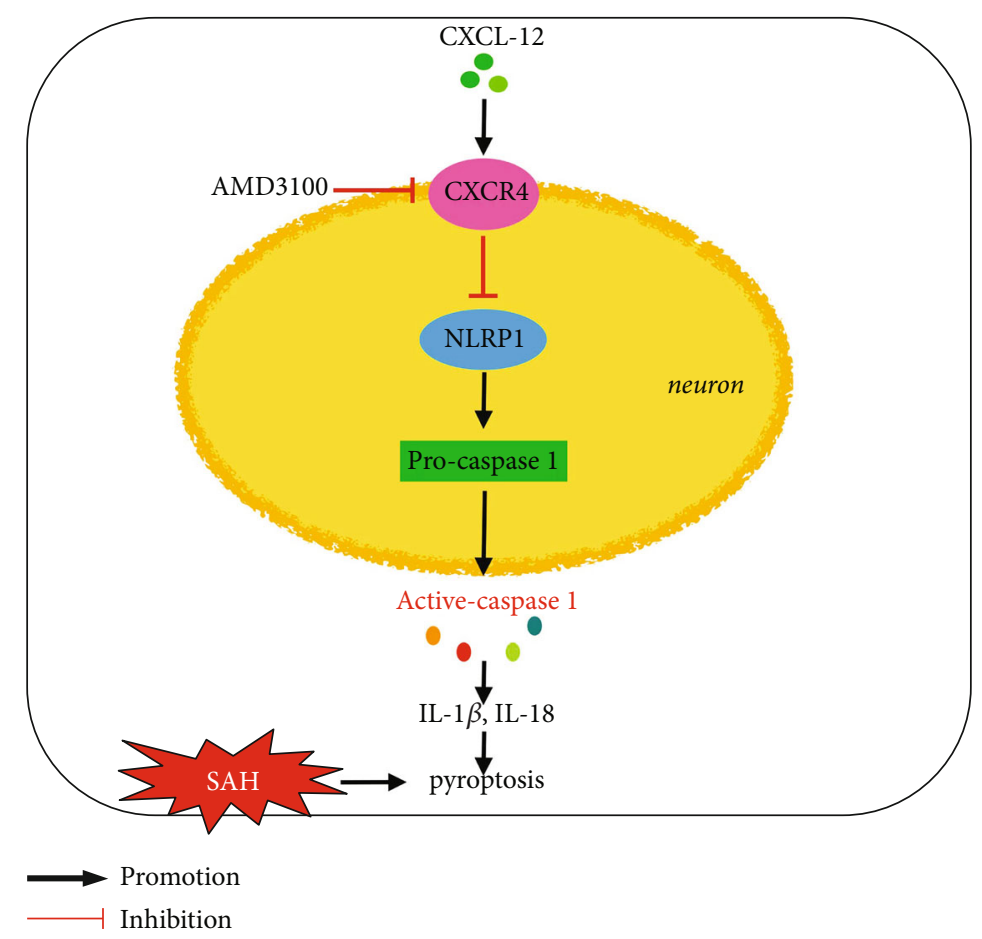

FIGURE 1: Schematic summary. Rh-CXCL-12 attenuates neuronal pyroptosis via the CXCR4/NLRP1 signaling pathway in a rat model of SAH.

[6]; all of which initiate a variety of pathophysiological events, such as oxidative stress, neuroinflammation, bloodbrain barrier dysfunction, apoptosis, and pyroptosis [7, 8]. Pyroptosis induced by SAH has been considered a critical devastating pathophysiological process in EBI after SAH [9].

Inflammasome is a multiprotein complex, which is a significant factor in the mechanism of pyroptosis [10]. As the first member of the NACHT Leucine-rich (NLR) family, NACHT Leucine-rich-repeat protein 1 (NLRP1) is mainly expressed on neurons and microglia [11]. After the assembly of NLRP1 inflammasome, cleaved caspase-1 (CC-1) is activated, and pro-IL-1 $\beta$ and pro-IL-18 are converted into IL$1 \beta$ and IL-18, which regulate pyroptosis. Previous studies have found that CC-1 was highly expressed in the wall of human brain aneurysms and was overexpressed in ruptured aneurysm tissue, indicating that pyroptosis was involved in the occurrence of SAH [12]. Other studies have found that the levels of NLRP1, ASC, and CC-1 in the cerebrospinal fluid of SAH patients are higher than the control group, which were associated with poor prognosis. Therefore, high level of NLRP1 is an independent risk factor for unfavourable prognosis after SAH [13].

Cysteine-X-cysteine chemokine ligand 12 (CXCL-12) is an inflammatory chemokine derived from bone marrow mesenchymal stem cells and belongs to the CXC chemokine family. CXC chemokine receptor type 4 (CXCR4) is one of the seven transmembrane G-protein-coupled receptors that mediates the transmembrane signaling of CXCL-12. CXCL-12 and CXCR4 are abundant and widely expressed in the central nerves system (CNS), playing important roles in neurogenesis and contributing to the neuronal development [14]. CXCL-12 is known to be expressed in neurons, glial cells, endothelial cells, and meningeal cells [15]. Previ- ous studies have shown that CXCL-12 is upregulated in the penumbra after stroke, reduces neurological deficits, and promotes angiogenesis [16-18]. CXCR4 is reportedly expressed in neurons, astrocytes, microglia, and ependymal cells [19]. Recent studies have demonstrated that CXCR4 is involved in the inflammatory response and exerts neuroprotective effects after ischemic stroke [20, 21]. Roosen et al. have suggested that CXCR4 reduces the pyroptosis of LPSstimulated BV2 cells [22]. Other studies have reported that CXCL-12 significantly reduces the levels of IL-18, IL-1 $\beta$, TNF-a, NLRP3, ASC, and CC-1 in a spinal cord injury model [23]. However, no research has been investigated to explore the role of CXCR4 and the underlying mechanism after SAH.

In the present study, we hypothesized that rh-CXCL-12 would attenuate neuronal prognosis by inhibiting NLRP1 and improve neurological impairment. Moreover, these beneficial effects were at least in part via regulation of CXCR4/NLRP1 signaling pathway after SAH in rats (Figure 1).

\section{Materials and Methods}

2.1. Animals. Adult male Sprague-Dawley rats (weighting 280-330 g, $n=136$ ) obtained from Guizhou Laboratory Animal Engineering Technology Center (China) were used in this project. All animals were kept in a room with controlled humidity $(60 \pm 5 \%)$ and constant temperature $\left(25 \pm 1^{\circ} \mathrm{C}\right)$, and remained in a $12 \mathrm{~h}$ light and dark cycle and with ad libitum access to food and water.

2.2. SAH Model. The SAH model was performed in rats using a modified endovascular perforation model as 
previously described [24]. Induction of anesthesia in rats was achieved using 4\% isoflurane and was maintained using $2.5 \%$ isoflurane. After intubation, the mice were placed in the supine position and connected to the rodent ventilator to breathe medical air (70\%) and oxygen (30\%). The heart rate, respiration, skin color, and pedal reflex assessment were assessed every five minutes during the operation to confirm anesthesia status and prevent distress. After exposing the carotid artery and its bifurcation, a 4-0 sharp single nylon thread suture was inserted from the external carotid artery into the left internal carotid artery to the anterior and middle cerebral artery bifurcation. The nylon suture was withdrawn immediately, and isoflurane was reduced to $1.5 \%$. After the operation, the endotracheal tube was removed and the animals were placed in the heating chamber $\left(37.5^{\circ} \mathrm{C}\right)$ to recover. Animals in the sham group underwent the same procedure, but without arterial wall puncture.

2.3. SAH Grading. The degree of SAH was assessed according to the SAH grading scale system at $24 \mathrm{~h}$ after $\mathrm{SAH}$ as previously described [25]. Rats with a score of 8 or less were excluded from the current study.

2.4. Experimental Design. Four separate experiments were performed as follows.

2.4.1. Experiment 1. To determine the time course of endogenous CXCL-12, CXCR4, and NLRP1 protein level expression in the sham group and each group after SAH. The rats were randomly divided into six groups ( $n=6 /$ group): sham, SAH-6h, SAH-12 h, SAH-24h, SAH-48 h, and SAH$72 \mathrm{~h}$. Western blot was performed to assess the protein levels of CXCL-12, CXCR4, and NLRP1 in the ipsilateral (left) hemisphere cerebral cortex. Additionally, the cellular localization of CXCR4 with calcium-binding adaptor molecule 1 (Iba-1), neuronal nucleus antigen (NeuN), and glial fibrillary acidic protein (GFAP) was evaluated using double immunofluorescence staining in the sham and $\mathrm{SAH}-24 \mathrm{~h}$ group ( $n=2$ /group).

2.4.2. Experiment 2. To evaluate the neuroprotective effects of CXCL-12 on short-term neurological outcomes after $\mathrm{SAH}$, rats were randomly assigned to five groups $(n=6$ (group): sham, SAH+vehicle (sterile distilled water), $\mathrm{SAH}$ +CXCL-12 $(5 \mu \mathrm{g} / \mathrm{kg})$, SAH+CXCL-12 $(15 \mu \mathrm{g} / \mathrm{kg})$, and SAH +CXCL-12 (45 $\mu \mathrm{g} / \mathrm{kg})$. CXCL-12 was administered intranasally (i.n.) at $1 \mathrm{~h}$ after SAH. The SAH grading score, neurobehavioral test (including modified Garcia test and beam balance test), and brain water content were assessed at $24 \mathrm{~h}$ after SAH in all groups. The best dose of CXCL-12 was selected based on the short-term neurological outcomes and brain water content results, which was also used for the mechanism experiments.

2.4.3. Experiment 3. To explore the effects of CXCL-12 on neuronal degeneration and pyroptosis at $24 \mathrm{~h}$ after $\mathrm{SAH}$, rats were randomly assigned to three groups ( $n=4$ /group): sham, SAH+vehicle (sterile distilled water), and $\mathrm{SAH}$ +CXCL-12 (optimal dose). Fluoro-Jade C (FJC) staining was performed, and double immunofluorescence staining was used to evaluate the CC- 1 and IL-18 expression on neurons in the perihemorrhagic area at $24 \mathrm{~h}$ after SAH. Numbers of FJC-positive cells, CC-1-positive neurons, and IL18-positive neurons were counted. Brain samples of these three groups were shared with experiment 4 .

2.4.4. Experiment 4. To explore the underlying mechanism of the CXCR4/NLRP1 signaling pathway-mediated antipyroptosis effects after SAH, the selective CXCR4 inhibitor, AMD3100, was administered intraperitoneally (i.p.) at $1 \mathrm{~h}$ before SAH. Rats were randomly assigned to five groups ( $n=6$ /group): sham, SAH+vehicle (sterile distilled water, i.n.), SAH+CXCL-12, SAH+CXCL-12+AMD3100, and $\mathrm{SAH}+\mathrm{CXCL}-12+\mathrm{PBS}$ (vehicle of AMD3100). The ipsilateral (left) hemisphere of each group was collected for Western blot analysis ( $n=6$ /group) after neurological performance, and $\mathrm{SAH}$ grades were evaluated at $24 \mathrm{~h}$ after $\mathrm{SAH}$.

2.5. Drug Administration. CXCL-12 or vehicle was given via intranasal administration at $1 \mathrm{~h}$ after $\mathrm{SAH}$ as previously described [26]. Animals were placed in the supine position and were administered $1.5 \%$ isoflurane anesthesia. A total volume of $20 \mu \mathrm{L}$ of vehicle (sterile distilled water) or CXCL-12 (MedChem Express, NJ, USA) at three different doses $(5 \mu \mathrm{g} / \mathrm{kg}, 15 \mu \mathrm{g} / \mathrm{kg}$, and $45 \mu \mathrm{g} / \mathrm{kg})$, with $5 \mu \mathrm{L}$ administered every 5 minutes, alternating between the right and left nares. AMD3100 was diluted in PBS and administered intraperitoneally (i.p.) at $1 \mathrm{~h}$ before $\mathrm{SAH}$.

2.6. Assessment of Short-Term Neurological Performance. The short-term neurobehavioral outcomes were assessed blindly using the 18 points modified Garcia scoring system and the 4 points beam balance test at $24 \mathrm{~h}$ after SAH as previously described [27]. Higher scores indicated better neurological function.

2.7. Brain Water Content. Brain edema was assessed by measuring brain water content using the wet-dry method as previously described [8]. The rats were euthanized at $24 \mathrm{~h}$ after $\mathrm{SAH}$, and the brains were quickly removed and separated into four parts (right hemisphere, left hemisphere, cerebellum, and brain stem). Afterwards, each part of the brain was weighed immediately to obtain the wet weight and then placed into an oven for $72 \mathrm{~h}$ at $100^{\circ} \mathrm{C}$. The dried brain was weighed again. The percentage of brain water content was calculated as follows: (wet weight - dry weight)/wet weight $\times 100 \%$.

2.8. Immunofluorescence Staining. The rats were deeply anesthetized (5\% isoflurane) and euthanized via transcardiac perfusion with $100-150 \mathrm{~mL}$ of precooled PBS $\left(4^{\circ} \mathrm{C}\right)$ and $100 \mathrm{~mL}$ of $10 \%$ formalin. Whole brains were rapidly collected and fixed in $10 \%$ formalin $\left(4^{\circ} \mathrm{C}, 24 \mathrm{~h}\right)$, followed by dehydration with $30 \%$ sucrose $\left(4^{\circ} \mathrm{C}, 72 \mathrm{~h}\right)$. Brain samples were embedded in OCT (Scigen Scientific, Gardena, CA, USA), and then frozen at $-80^{\circ} \mathrm{C}$. The brains were sliced into $10 \mu \mathrm{m}$ thick coronal brain sections using a cryostat (CM3050S, Leica Microsystems, Bannockburn, Germany) and then mounted onto normal poly-L-Lysine-coated slides. The slices were washed with $0.01 \mathrm{M}$ of PBS three times for 5- 


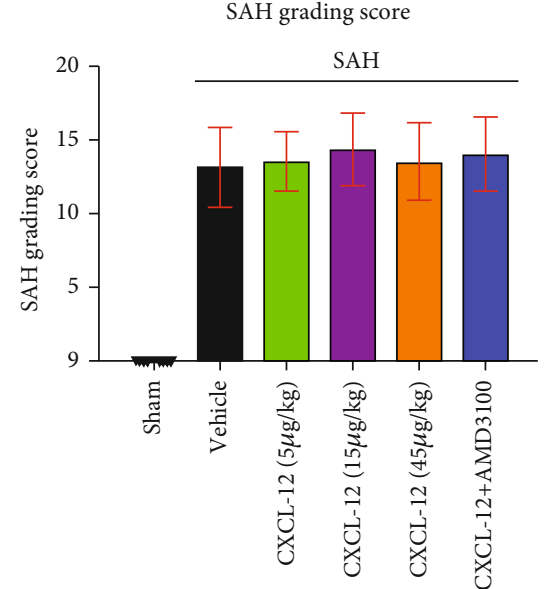

(a)

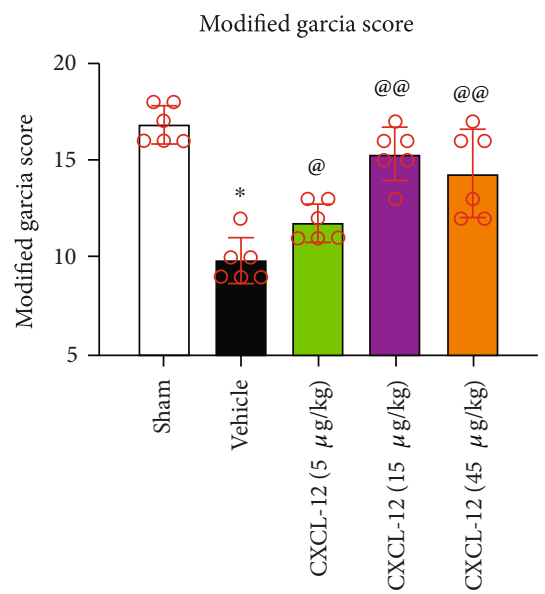

SAH

(c)

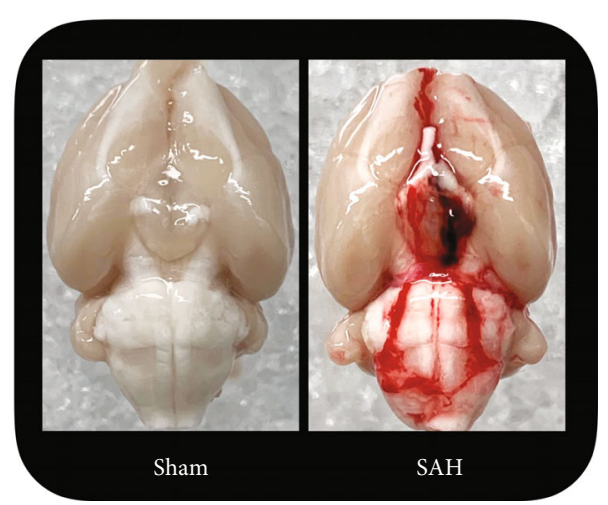

(e)

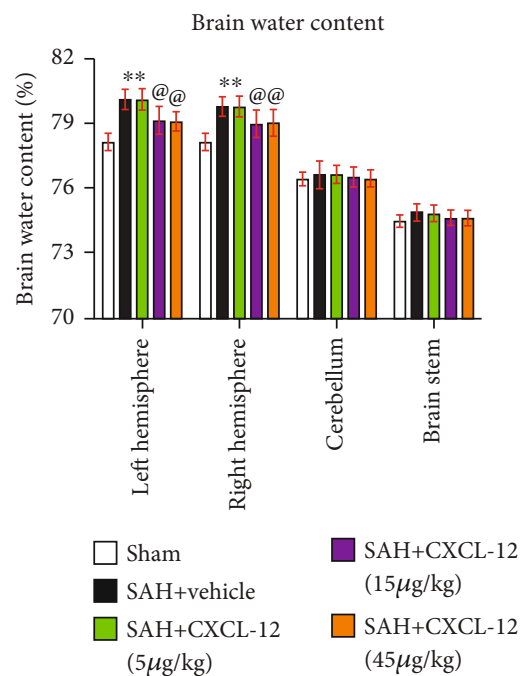

(b)

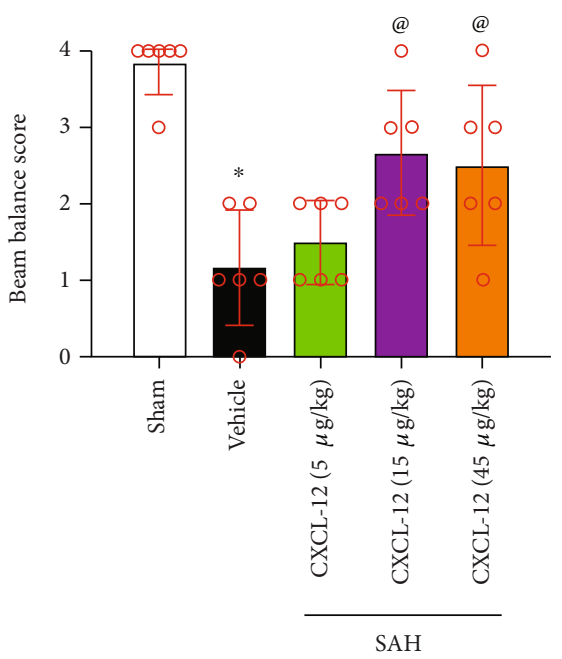

(d)

FIGURE 2: Intranasal administration of rh-CXCL-12 improved short-term neurological outcome and attenuated brain edema at $24 \mathrm{~h}$ after SAH. (a) SAH grading score of each group. (b) Quantification of brain water content in the left hemisphere, right hemisphere, cerebellum, and brain stem at $24 \mathrm{~h}$ after SAH. (c, d) Intranasal administration of rh-CXCL-12 improved neurological performance on the modified Garcia and beam balance test at $24 \mathrm{~h}$ after SAH. (e) Representative pictures of the brain in the sham and SAH groups (subarachnoid blood clots were mainly presented around the circle of Willis). Vehicle: sterile distilled water. Data were presented as mean \pm SD. $n=6$ per group. ${ }^{*} P<0.05$ vs. sham group; ${ }^{\circledR} P<0.05,{ }^{@} P<0.01$ vs. SAH+vehicle group. 


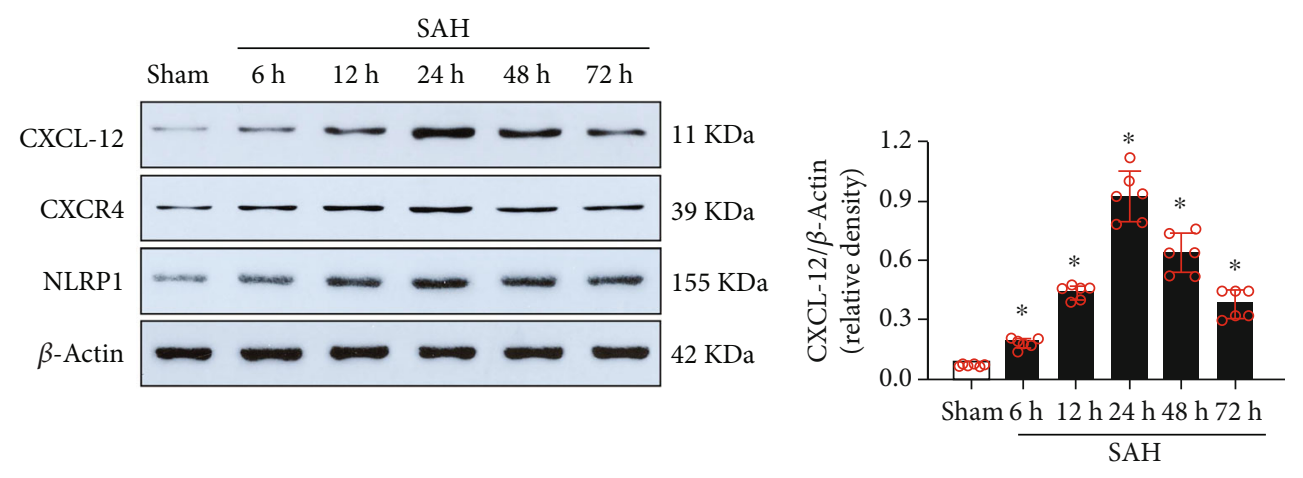

(a)

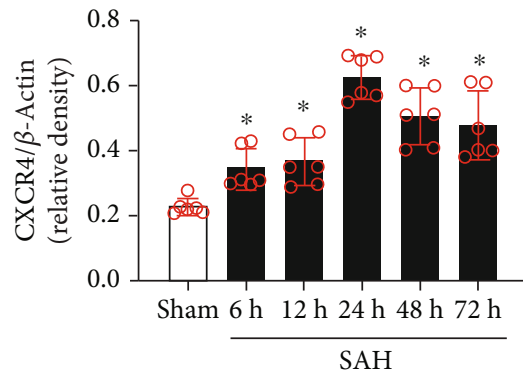

(c) (b)

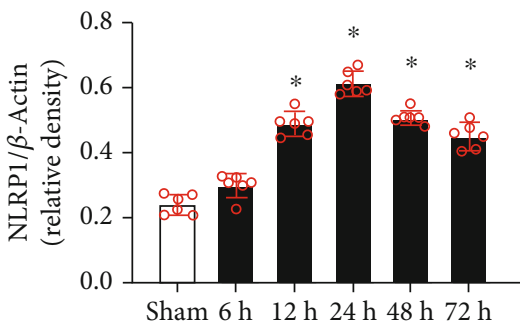

(d)

FIGURE 3: Time course of CXCL-12, CXCR4, and NLRP1, as well as cellular localization of CXCR4 receptor after SAH. (a) Representative western blot bands of time course and densitometric quantification of CXCL-12, CXCR4, and NLRP1 (a-d) in the ipsilateral hemisphere after SAH. Data were presented as mean \pm SD. ${ }^{*} P<0.05$ vs. sham group; $n=6$ per group.

$10 \mathrm{~min}$ and then incubated in $0.3 \%$ Triton $\mathrm{X}-100$ in $0.01 \mathrm{M}$ of PBS for $10 \mathrm{~min}$ at room temperature. After being blocked with $5 \%$ donkey serum in $0.01 \mathrm{M}$ of PBS for $2 \mathrm{~h}$ at room temperature, the sections were incubated overnight at $4^{\circ} \mathrm{C}$ with the following primary antibodies: anti-Iba-1 (1:200, Abcam), anti-NeuN (1:200, Abcam), anti-GFAP $(1: 200$, Abcam), anti-CC-1 (1:100, Santa Cruz Biotechnology), anti-IL-18 (1:200, Abcam), and anti-CXCR4 (1:200, Abcam). Next, the slices were incubated with fluorescenceconjugated secondary antibodies $(1: 500)$ for $1 \mathrm{~h}$ at room temperature. The slides were visualized and photographed using a fluorescence microscope. To assess neuronal pyroptosis levels, the number of CC-1-positive neurons and IL-18positive neurons was identified and counted in three different fields from the left basal cortex of five random coronal sections of each rat. The positive cells were quantified under a microscopic field of 200x magnification, and data were expressed as cells/field.

2.9. FJC Staining. To detect neuronal degeneration, FJC staining was performed using the FJC Ready-to-Dilute Staining Kit (Biosensis, USA) according to manufacturer's protocol. The stained slices were observed and photographed under a fluorescence microscope and analyzed by Leica Application Suite software. The brains of four rats per group were counted from 5 fields per brain in the per lesion region for quantification analysis. The FJC-positive cells were quantified under a microscopic field of $200 \mathrm{x}$ magnification, and data were expressed as cells/field.
2.10. Western Blot Analysis. At $24 \mathrm{~h}$ after SAH, rats were deeply anesthetized (5\% isoflurane) and transcardially perfused with chilled PBS, followed by decapitation. The brain sections were separated into ipsilateral and contralateral hemispheres. The ipsilateral hemisphere brain tissues were snap frozen in liquid nitrogen and stored in $\mathrm{a}-80^{\circ} \mathrm{C}$ freezer for storage until used. Brain samples were homogenized in RIPA lysis buffer with protease inhibitor for $15 \mathrm{~min}$ and then centrifuged at $14,000 \mathrm{~g}\left(4^{\circ} \mathrm{C}, 30 \mathrm{~min}\right)$. The supernatant was collected, and protein concentration was measured by detergent compatible assay (DC Protein Assay, Bio-Rad Laboratories). Equal amounts of protein were loaded onto the $10 \%$ SDS-PAGE gel for electrophoresis and then transferred onto nitrocellulose membranes. The membranes were blocked with 5\% nonfat blocking grade milk for $2 \mathrm{~h}\left(37^{\circ} \mathrm{C}\right)$ and incubated overnight at $4^{\circ} \mathrm{C}$ with the following primary antibodies: anti-CXCR4 (1:1000, Abcam), anti-CXCL-12 (1:1000, Abcam), antiNLRP1 ( $1: 1000$, Abcam), anti-CC-1 (1:1000, Cell Signaling Technology), anti-IL-18 (1:1000, Abcam), anti-IL-1 $\beta$ ( $1: 1000$, Abcam), and anti- $\beta$-actin ( $1: 5000$, Santa Cruz Biotechnology). The membranes were incubated with the appropriate peroxidase-conjugated secondary antibodies (1:5000, Santa Cruz) for $1 \mathrm{~h}$ at $37^{\circ} \mathrm{C}$. The bands were then visualized with the ECL Plus chemiluminescence reagent kit (Amersham Bioscience, Pittsburgh, PA) and quantified with the ImageJ software (ImageJ 1.5, NIH, USA).

2.11. Statistical Analysis. Statistical analysis was performed using GraphPad Prism 7 (Graph Pad Software, San Diego, CA, USA). All data were presented as mean \pm SD. One-way 


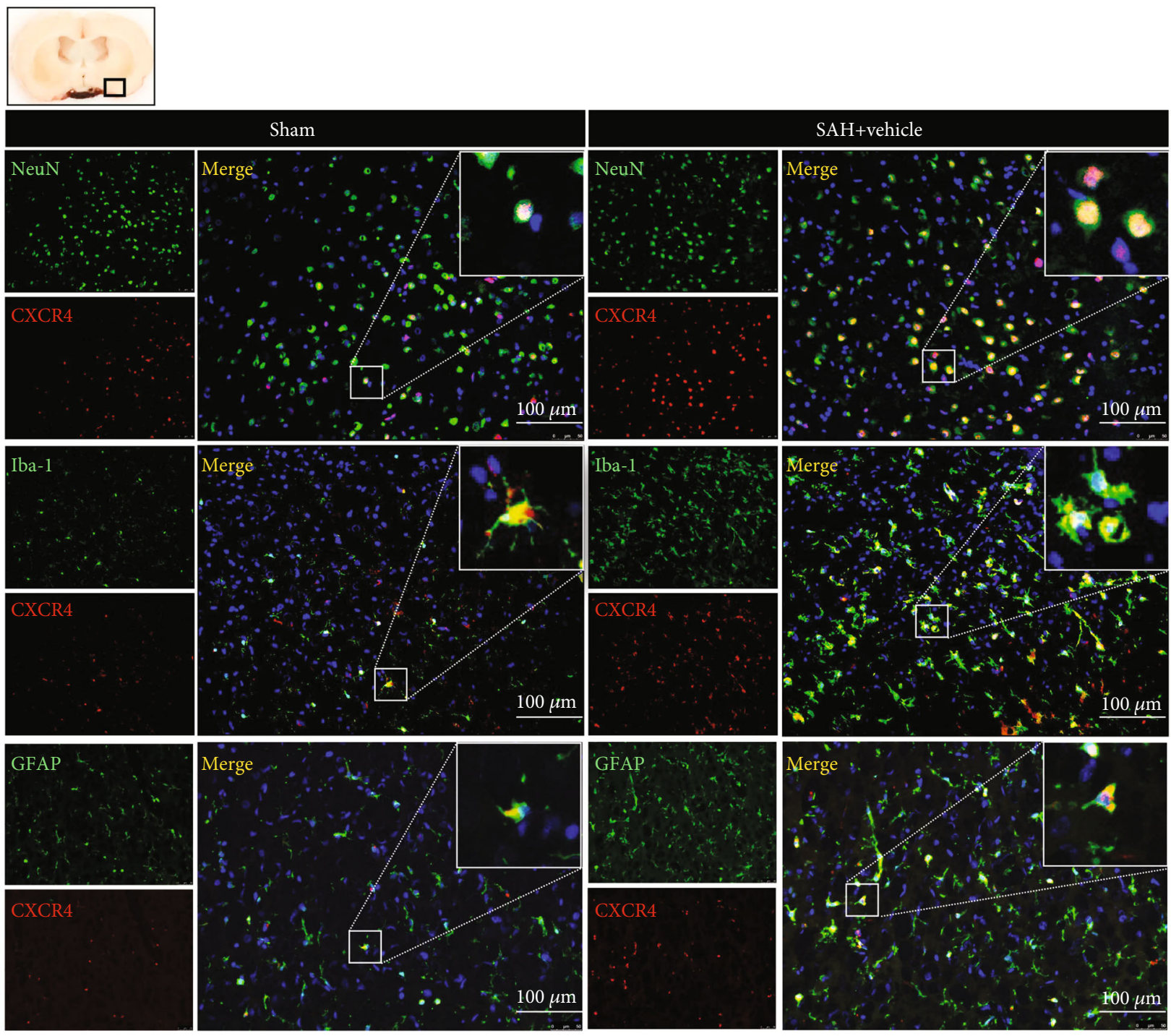

FIGURE 4: Coimmunofluorescence staining of CXCR4 (red) on neurons (NeuN, green), microglia (Iba-1, green), and astrocytes (GFAP, green) in the ipsilateral basal cortex at $24 \mathrm{~h}$ after SAH. Cell nuclei were counterstained with DAPI (blue). A small black square within the coronal section of the brain indicated the location of where the immunofluorescence staining images were taken. $n=2$ per group. Scale bar $=100 \mu \mathrm{m}$.

ANOVA followed by Tukey's post hoc test was used for comparison among multiple groups. Two-way ANOVA was used to analyze the long-term neurobehavioral results. $P<0.05$ was considered statistically significant.

\section{Results}

3.1. Mortality and SAH Grading Score. Of the 136 rats used, 112 rats underwent SAH induction. Of which, 15 (14.56\%) rats died within $24 \mathrm{~h}$ after SAH, and 9 rats were excluded from this project due to mild SAH. There was no mortality in the sham group. Subarachnoid blood clots were distributed around the circle of Willis and ventral brain stem after $\mathrm{SAH}$ induction, with a significant difference from the sham group (Figure 2(e)). The average SAH grading scores among all SAH groups showed no significant differences (Figure 2(a)).
3.2. Expression Levels of Endogenous CXCL-12, CXCR4, and NLRP1 and Colocalization of CXCR4 with Neurons, Microglia, and Astrocytes after SAH. As shown in Figure 3, the results of Western blotting showed that the endogenous protein expression levels of CXCL-12, CXCR4, and NLRP1 increased in a time-dependent manner, and peaked at $24 \mathrm{~h}$ after SAH when compared to the sham group $(P<0.05)$. Coimmunofluorescence staining of Iba-1, NeuN, and GFAP with CXCR4 showed that CXCR4 was expressed on neurons, microglia and astrocytes within cortices in the sham group and in the perihemorrhagic area at $24 \mathrm{~h}$ after $\mathrm{SAH}$ (showed as Figure 4).

3.3. Intranasal Administration of Exogenous rh-CXCL-12 Improved Short-Term Neurobehavioral Dysfunctions and Attenuated Brain Edema at $24 \mathrm{~h}$ after SAH. The brain water content in the left and right hemisphere was significantly 

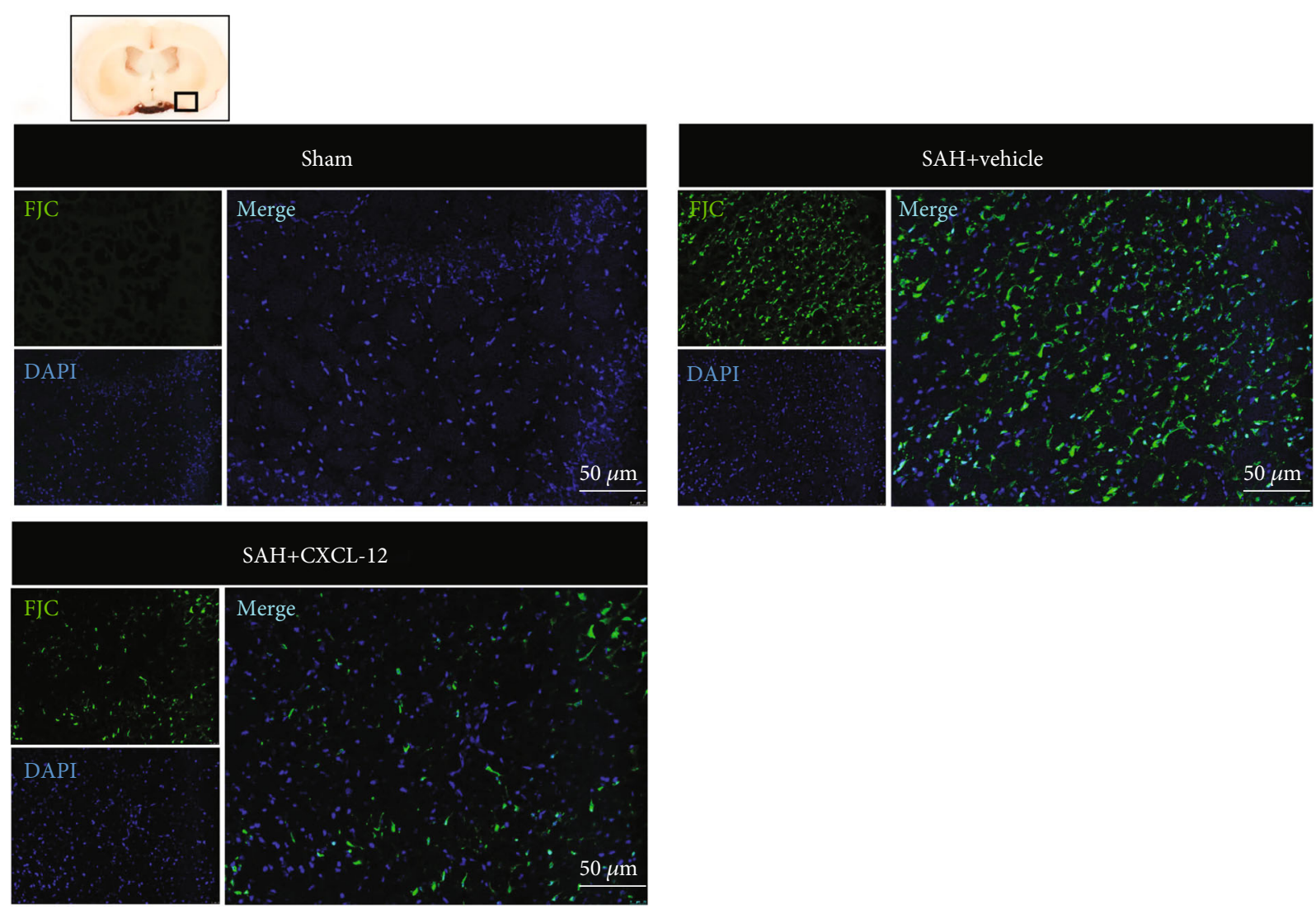

(a)

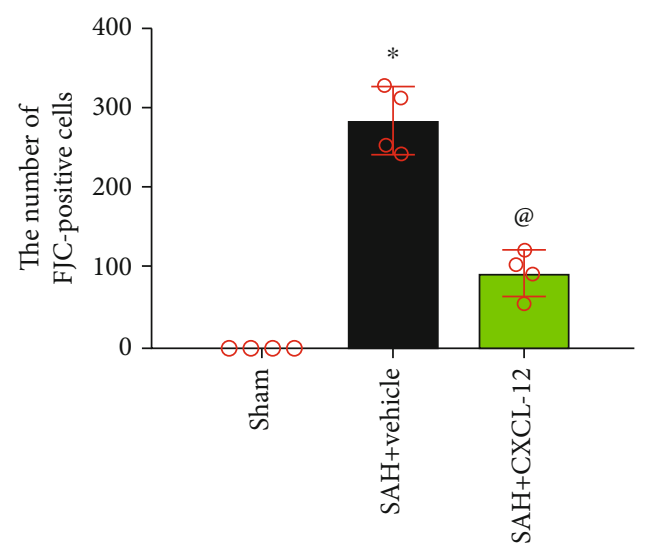

(b)

FIgURE 5: Intranasal administration of rh-CXCL-12 attenuated neuronal degeneration at 24h after SAH. (a) Representative immunofluorescence staining of FJC-positive cells in the ipsilateral basal cortex at $24 \mathrm{~h}$ after SAH. Green indicated FJC-positive staining, and blue indicated DAPI-positive nuclear staining. A small black square within the coronal section of the brain indicated the location of where the immunofluorescence staining images were taken. (b) Quantitative analysis of FJC-positive cells. $n=4$ per group. Vehicle: sterile distilled water. Data were represented as mean $\pm \mathrm{SD} .{ }^{*} P<0.05$ vs. sham group; ${ }^{\circledR} P<0.05$ vs. SAH+vehicle group.

increased in the SAH+vehicle and SAH+rh-CXCL-12 $(5 \mu \mathrm{g} / \mathrm{kg})$ group, which was significantly reduced by the administration of rh-CXCL-12 at doses of $15 \mu \mathrm{g} / \mathrm{kg}$ and $45 \mu \mathrm{g} / \mathrm{kg}(P<0.05$, Figure $2(\mathrm{~b}))$. Brain water contents in the cerebellum and brain stem were not significantly different between the sham and SAH groups. The neurobehavioral outcomes of modified Garcia and beam balance were significantly reduced at $24 \mathrm{~h}$ after $\mathrm{SAH}$ in the SAH+vehicle and SAH+rh-CXCL-12 (5 $\mu \mathrm{g} / \mathrm{kg})$ groups. However, administration of rh-CXCL-12 (15 $\mu \mathrm{g} / \mathrm{kg})$ and rh-CXCL-12 $(45 \mu \mathrm{g} / \mathrm{kg})$ significantly improved the neurological scores at $24 \mathrm{~h}$ after SAH $(P<0.05$, Figures $2(\mathrm{c})$ and $2(\mathrm{~d}))$. Based on these results, the optimal dose of Rh-CXCL-12 was $15 \mu \mathrm{g} / \mathrm{kg}$, which was used for the mechanistic studies. 


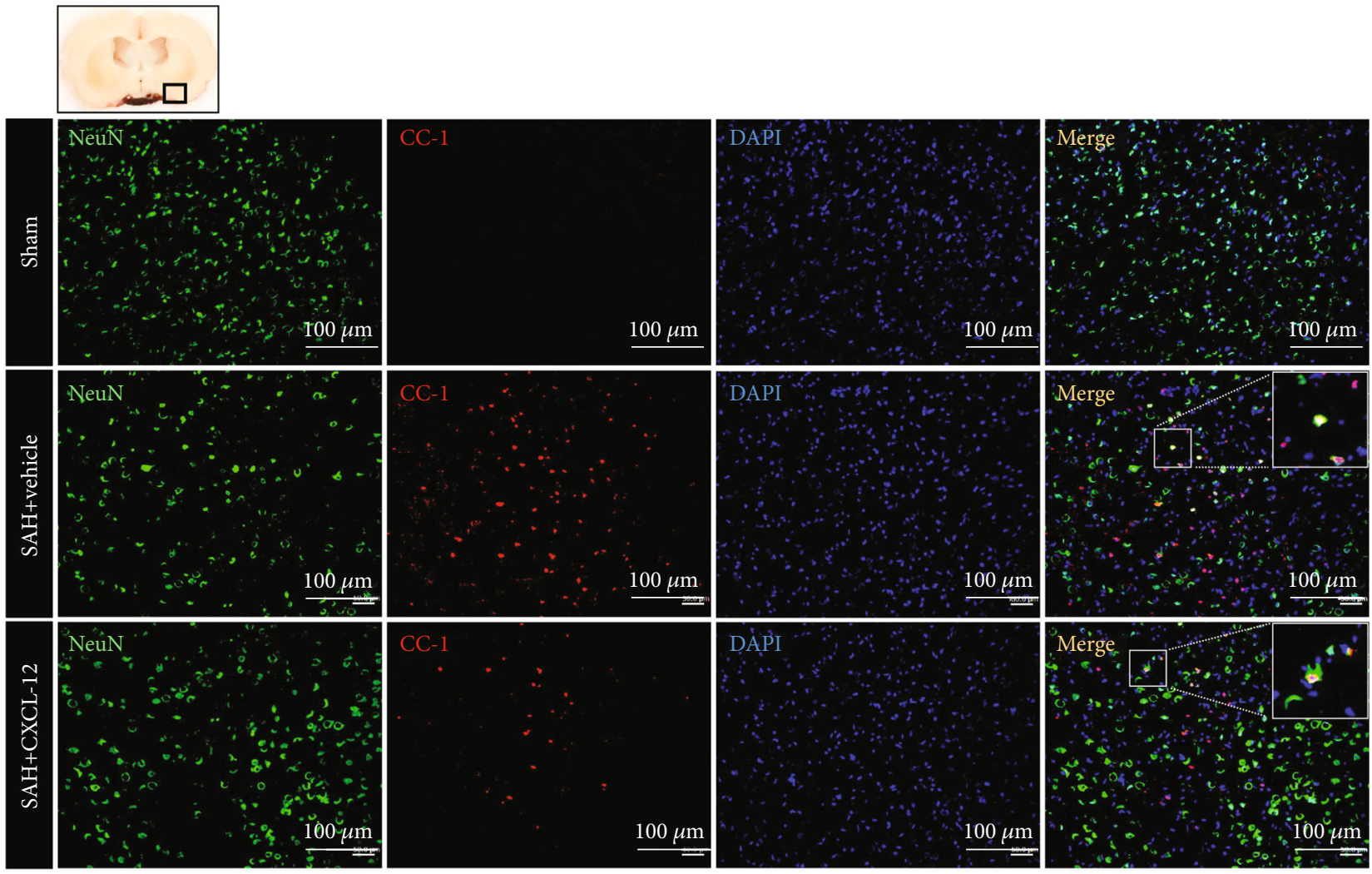

(a)

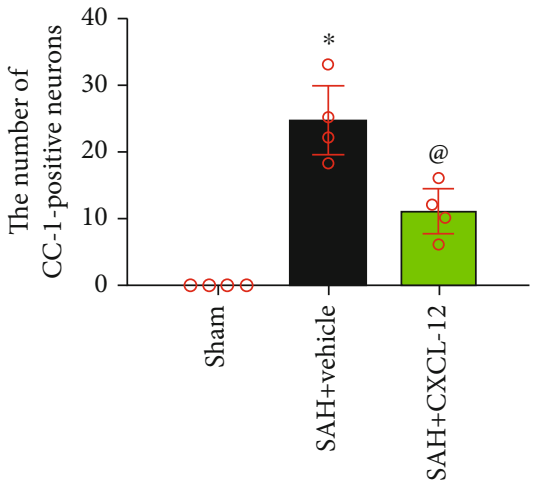

(b)

Figure 6: Intranasal administration of rh-CXCL-12 attenuated CC-1-positive neurons after SAH. (a) Representative immunofluorescence staining of CC-1-positive neurons in the ipsilateral basal cortex at $24 \mathrm{~h}$ after SAH. Green indicated CC1-positive staining, red indicated neurons, and blue indicated DAPI-positive nuclear staining. A small black square within the coronal section of the brain indicated the location of where the immunofluorescence staining images were taken. (b) Quantitative analysis of CC-1-positive neurons. $n=4$ per group. Vehicle: sterile distilled water. Data were represented as mean $\pm \mathrm{SD}$. ${ }^{*} P<0.05$ vs. sham group; ${ }^{@} P<0.05$ vs. SAH+vehicle group.

3.4. Rh-CXCL-12 Reduced Neuronal Degeneration and Pyroptosis at $24 \mathrm{~h}$ after SAH. FJC staining showed that the FJC-positive cells were reduced after rh-CXCL-12 administration (Figure 5). Double-immunofluorescence staining of neurons with CC-1 and IL-18 were performed to evaluate neuronal pyroptosis in the ipsilateral basal cortex at $24 \mathrm{~h}$ after SAH. Compared with the sham group, rats in the $\mathrm{SAH}+$ vehicle group showed an increase in CC-1-positive neurons and IL-18-positive neurons. Rats treated with rhCXCL-12 had fewer CC-1-positive neurons and IL-18positive neurons at $24 \mathrm{~h}$ after SAH (Figures $6(\mathrm{a})$ and $7(\mathrm{a})$ ).
Quantitative analysis showed that rh-CXCL-12 administration significantly reduced the number of CC-1-positive neurons and IL-18-positive neurons (Figures 6(b) and 7(b)).

3.5. Administration of rh-CXCL-12 Attenuated Neuronal Pyroptosis through the CXCR4/NLRP1 Signaling Pathway at $24 \mathrm{~h}$ after $S A H$. Western blot results showed that the pathway-related proteins, CXCL-12, CXCR4, NLRP1, IL$1 \beta$, IL-18, and CC-1, were upregulated in the SAH+vehicle group at $24 \mathrm{~h}$ after $\mathrm{SAH}$ when compared with the sham group (Figures 8(a)-8(h)). Rh-CXCL-12 treatment further 


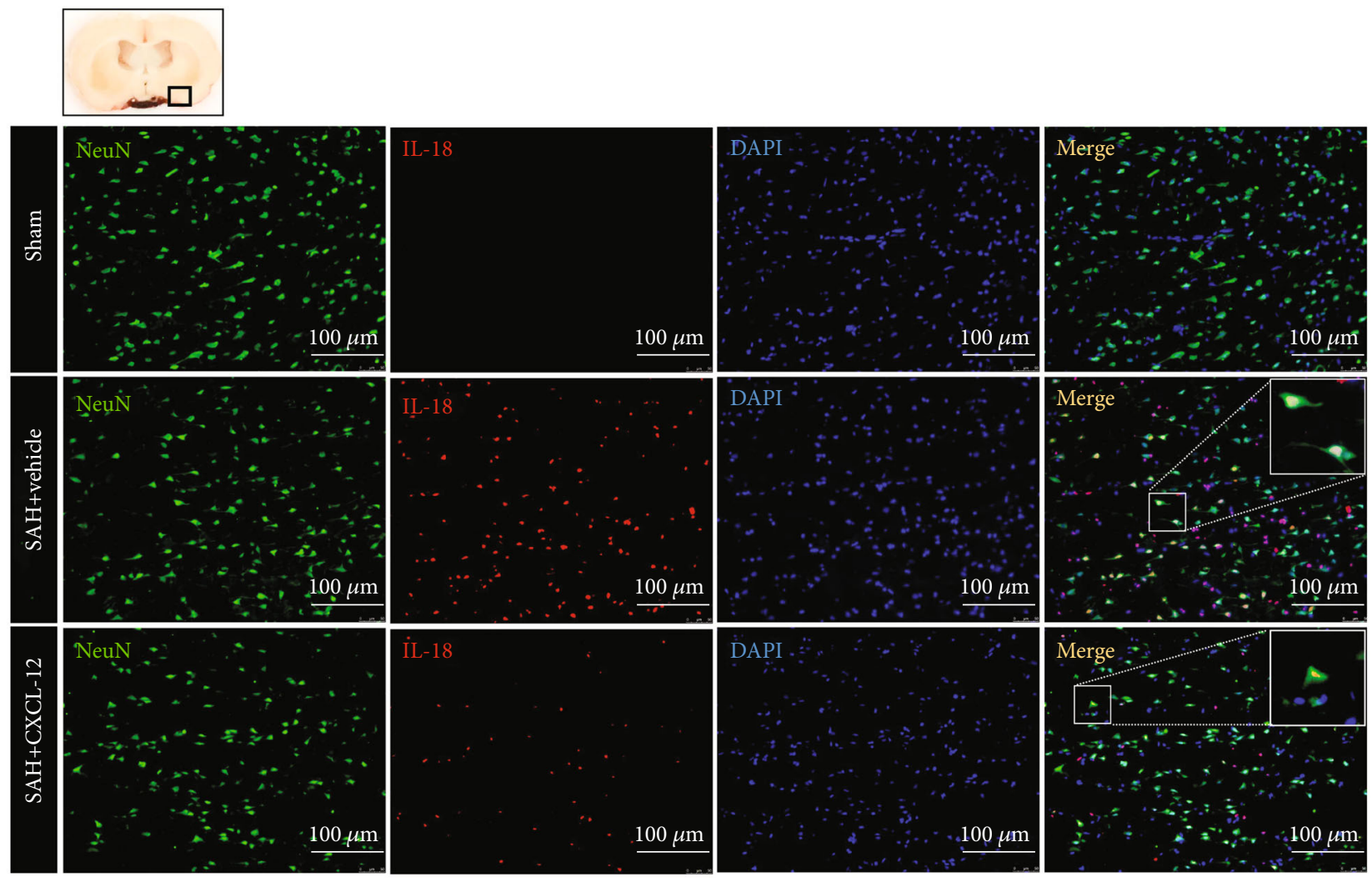

(a)

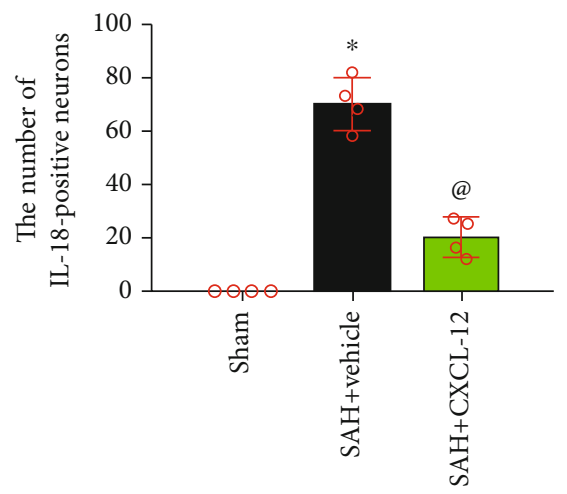

(b)

FIGURE 7: Intranasal administration of rh-CXCL-12 attenuated IL-18-positive neurons after SAH. (a) Representative immunofluorescence staining of IL-18-positive neurons in the ipsilateral basal cortex at $24 \mathrm{~h}$ after SAH. Green indicated IL-18-positive staining, red indicated neurons, and blue indicated DAPI-positive nuclear staining. A small black square within the coronal section of the brain indicated the location of where the immunofluorescence staining images were taken. (b) Quantitative analysis of IL-18-positive neurons. $n=4$ per group. Vehicle: sterile distilled water. Data were represented as mean $\pm \mathrm{SD}$. ${ }^{*} P<0.05$ vs. sham group; ${ }^{\circledR} P<0.05$ vs. SAH+vehicle group.

increased the expression levels of CXCL-12 and CXCR4, but decreased the expressions of NLRP1, IL-1 $\beta$, IL-18, and CC1, compared with the SAH+vehicle group (Figures 8(a)8(h)). The administration of AMD3100 reversed regulation of pathway-related proteins and the antipyroptosis effects of rh-CXCL-12 at $24 \mathrm{~h}$ after SAH (Figures $8(\mathrm{a})-8(\mathrm{~h})$ ).

\section{Discussion}

The present study was the first to investigate the neuroprotective effects of CXCL-12 and explore the potential underly- ing mechanisms after experimental SAH in rats. Our results demonstrated that (1) endogenous protein levels of CXCL12, CXCR4, and NLRP1 were increased and peaked at $24 \mathrm{~h}$ after SAH. The CXCR4 receptors were expressed on microglia, neuron, and astrocytes at $24 \mathrm{~h}$ after SAH. (2) Rh-CXCL12 improved short-term neurological deficits and ameliorated brain edema at $24 \mathrm{~h}$ after SAH. Furthermore, rhCXCL-12 treatment reduced the number of FJC-positive cells, CC-1-positive neurons, and IL-18-positive neurons in the perihemorrhagic area in the ipsilateral cerebral cortex; (3) administration of rh-CXCL-12 significantly increased 


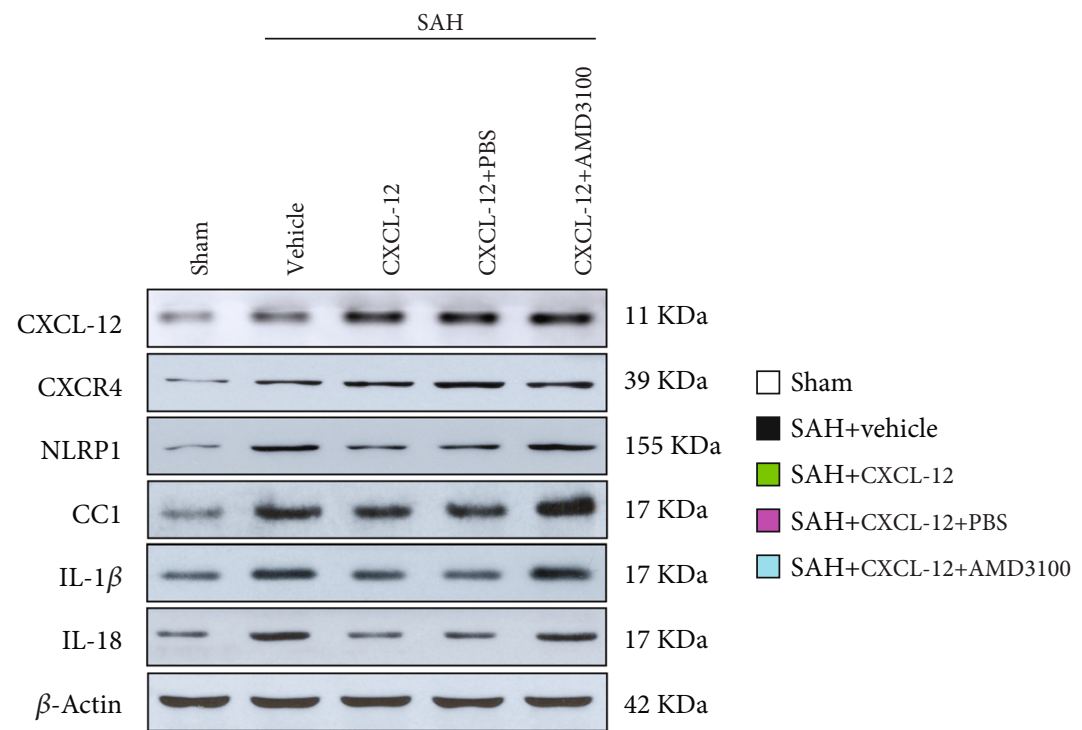

(a)

(b)

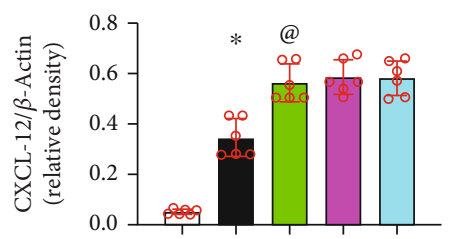

(c)

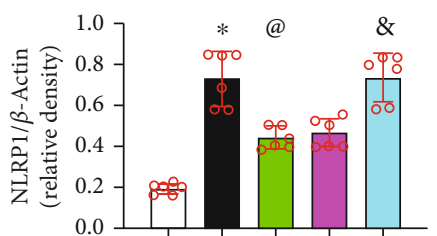

(e)

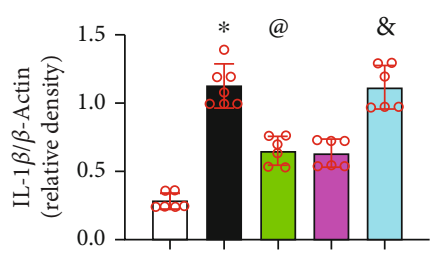

(g)

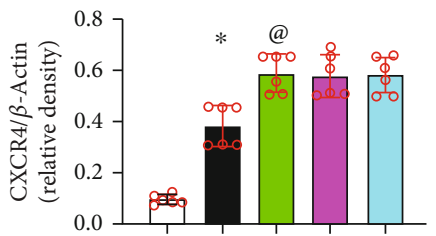

(d)

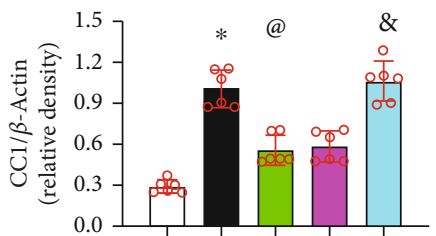

(f)

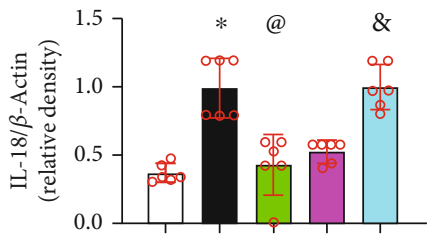

(h)

FIGURE 8: Effects of CXCR4 inhibitor on downstream proteins in the proposed signaling pathway with rh-CXCL-12 treatment at $24 \mathrm{~h}$ after SAH. (a) Representative Western blot bands of the proteins CXCL-12, CXCR4, NLRP1, IL-1 $\beta$, CC-1, and IL-18. (b-h) Quantitative analysis of the relative expression level of these proteins at $24 \mathrm{~h}$ after SAH. ${ }^{*} P<0.05$ vs. sham; ${ }^{\circledR} P<0.05$ vs. SAH+vehicle group; ${ }^{\circledR} P<0.05$ vs. SAH + PBS group; $n=6$ per group.

the expression levels of CXCL-12 and CXCR4, but decreased the expression of NLRP1, IL-1 $\beta$, IL-18, and CC-1; (4) CXCR4 inhibitor, AMD3100, reversed the antipyroptosis effects of rh-CXCL-12 and its effects on the CXCR4/NLRP1 signaling pathway. Taken together, our results showed that the activation of CXCR4 with rh-CXCL-12 may exert a neuroprotective effect and improve neurological function by reducing neuronal pyroptosis after $\mathrm{SAH}$, and these effects were at least in part via activation of the CXCR4/NLRP1 signaling pathway.
CXCR4, a chemokine receptor in the G protein-coupled receptor gene family, is widely found in the CNS and immune cells. It induces immune cell migration and nervous system development by binding to its ligand, CXCL- 12 . CXCR4 is involved in regulating the inflammatory response in CNS diseases, such as Alzheimer's disease [28], ischemic stroke [20], and Parkinson's disease [29]. Furthermore, some other studies have reported that CXCL-12/CXCR4 reduced neuronal apoptosis after traumatic brain injury and ischemic stroke $[15,30]$. In the present study, our results showed that 
the expression of exogenous CXCL-12 and CXCR4 increased at the early stage of SAH and peaked at $24 \mathrm{~h}$ after SAH. The increased expression of CXCL-12 and CXCR4 may explain its participation in the endogenous neuroprotective mechanisms after SAH, which were insufficient in overriding the injury. Furthermore, we found that CXCR4 colocalized with neurons, microglia, and astrocytes using the double immunofluorescence method, and the number of CXCR4-positive neurons, microglia, and astrocytes was significantly increased at 24 after SAH, which also indicated that CXCR4 was involved in neuronal pyroptosis in EBI after SAH.

The pathogenesis of SAH is varied, including neuroinflammation, apoptosis, autophagy, and pyroptosis [7, 8, 31, 32]. Musluman et al. investigated the association between apoptotic neurons in the petrosal ganglion 20 days after $\mathrm{SAH}$ and found petrosal ganglion ischemia caused neuronal apoptosis [33]. Pyroptosis, different than apoptosis, represents a form of cell death triggered by proinflammatory signal and associated with inflammation [34], which is seen primarily in inflammatory cells such as macrophages and may be triggered by bacterial or pathogen infections. This type of cell death has also been confirmed in traumatic brain injury and acute hemorrhagic stroke $[35,36]$. Pyroptosis is activated by two caspase-dependent pathways, including the typical caspase 1 and the atypical caspase 4/5/11 pathway [37]. The cardinal feature of pyroptosis is the requirement for caspase 1 activation. Inflammasome plays an important role in the typical caspase 1 pathway $[38,39]$. Caspase 1 is responsible for the maturation of proinflammatory cytokines such as IL- $1 \beta$ and IL-18 through inflammasomedependent pathways [40]. Inflammasomes are multimeric protein complexes; NLR family (NLRP1, NLRP3, NLRC4, NLRP6, and NLRP9) are major inflammasomes. It was shown that the NLRP3 protein is upregulated after SAH and peaked at $24 \mathrm{~h}$, along with the elevation of inflammatory factors, IL- $1 \beta$ and IL-18 [41]. The NLRP1 inflammasome, which has been reported to be primarily expressed in neurons and glial cells, is the first member that has been characterized among the NLR family. Among known inflammasomes, NLRP1, NLRP2, NLRP3, and AIM2 were widely studied in CNS disease, especially for NLRP1, which played a key role in SAH via-mediated neuronal pyroptosis [13]. Activation of NLRP1 inflammasome is essential for the regulation of proinflammatory cytokines, and overexpression of inflammatory factors can induce EBI after SAH $[13,42]$. Based on this mechanism and targeting, selective pharmacological molecule treatment with inhibition of the NLRP1 inflammasome could decrease neuronal injury after SAH. In the present study, our results showed that the expression of exogenous NLRP1 increased at the early stage of SAH. The increased NLRP1 expression indicated that the inflammasome is involved in the pathogenesis of SAH.

We then evaluated the effects of rh-CXCL-12 in the experimental SAH model. Our results showed that the intranasal administration of rh-CXCL-12, at a dose of $15 \mu \mathrm{g} / \mathrm{kg}$, reduced brain water content and improved short-time neurobehavioral outcomes, which were used as the best dose of rh-CXCL-12 in further experiment. Meanwhile, we dem- onstrated strong neuronal degeneration and pyroptosis, as evidenced by increased FJC-positive cells, CC-1-positive neurons, and IL-18-positive neurons at $24 \mathrm{~h}$ after SAH, which was consistent with previous investigations [42, 43]. Intranasal administration of rh-CXCL-12 reduced the number of FJC-positive cells, CC-1-positive neurons, and IL-18positive neurons. The results suggested that treatment of rhCXCL-12 could attenuate neuronal degeneration and pyroptosis after SAH.

We further investigated the underlying molecular mechanism of CXCL-12-induced antipyroptosis effects after SAH. It has been found that SDF-1 ameliorated NLRP3 inflammasome and pyroptosis in OA synoviocytes by activating AMPK signaling pathway [44]. In this study, we found that rh-CXCL-12 treatment significantly improved the modified Garcia and beam balance scores and increased the expression levels of CXCR4, but decreased the expression of inflammasome NLRP1 and inflammatory cytokines, IL-1 $\beta$, CC-1, and IL-18. Furthermore, CXCR4 inhibitor, AMD3100, reversed the neuroprotective effects of rhCXCL-12 by upregulating the levels of NLRP1, IL-1 $\beta$, CC1 , and IL-18. Taken together, rh-CXCL-12 attenuated neuronal degeneration and pyroptosis, which functioned, at least in part, by activating the CXCR4/NLRP1 signaling pathway after SAH.

This study has several limitations. First, this study focused primarily on the role of pyroptosis in the EBI phase and assessed the potential mechanisms of pyroptosis at $24 \mathrm{~h}$ after SAH. However, pyroptosis in delayed brain injury after SAH should also be investigated in future studies. Second, in addition to pyroptosis, the pathogenesis of $\mathrm{SAH}$ includes neuronal apoptosis, oxidative stress, and destruction of the blood-brain barrier; however, the present experiment only focused on pyroptosis. Therefore, the observation and study of other mechanisms should be elucidated in future experiments. Third, CXCR4 has other downstream signaling pathways, such as MEK/ERK and PI3K/Akt [45-47]. Therefore, more experiments are necessary to investigate the possible mechanisms of these signaling pathways and the neuroprotective effects of CXCL-12.

\section{Conclusions}

Our results demonstrated that the activation of CXCR4 with CXCL-12 improved short-term neurological deficits and attenuated neuronal pyroptosis in EBI after SAH in rats. The protective effects of CXCL-12 were at least in part through activation of the CXCR4/NLRP1 signaling pathway. Therefore, early administration of CXCL-12 may provide a therapeutic strategy against brain injury after SAH.

\section{Abbreviations}

ANOVA: Analysis of variance

AAALAC: Association for Assessment and Accreditation of Laboratory Animal Care International

BBB: Blood-brain barrier

CNS: Central nervous system

CXCL-12: Cysteine-X-cysteine chemokine ligand 12 
CXCR4: CXC, chemokine receptor type 4

EBI: $\quad$ Early brain injury

GFAP: Glial fibrillary acidic protein

i.n: Intranasally

i.p: Intraperitoneal

Iba-1: $\quad$ Ionized calcium-binding adaptor molecule 1

IL-1 $\beta$ : Interleukin-1 beta

NeuN: Neuronal nuclei antigen

SAH: Subarachnoid hemorrhage

SD: $\quad$ Standard deviation.

\section{Data Availability}

The data support the findings of this study and are available from the corresponding author upon reasonable request.

\section{Ethical Approval}

All animal experimental procedures were approved by the Guizhou Medical University and performed in accordance with the guidelines of the National Institutes of Health on the care and use of animals. Animals were housed in an Association for Assessment and Accreditation of Laboratory Animal Care International- (AAALAC-) approved animal quarters in Guizhou Medical University.

\section{Consent}

Consent for publication is not necessary.

\section{Conflicts of Interest}

The authors declare that they have no conflict of interest.

\section{Authors' Contributions}

This study was designed by RG, LW, GZ, and XH. The experiments were completed by RG, LW, XH, and GZ. $\mathrm{XH}, \mathrm{RG}$, and $\mathrm{CL}$ performed statistical analysis. XH, GZ, and LW finished writing the manuscript. GZ provided supervision and final check. All authors read the final version of this paper and approved it. Ran $\mathrm{Gu}$ and $\mathrm{Lu}$ Wang contributed equally to this work.

\section{Acknowledgments}

A preprint has previously been published [48]. This study was supported by grants H201654 and GSWS2019080 from Jiangsu Provincial Health and Family Planning Commission of China to Dr. G. Zuo; grant QiankeheZhicheng [2021] normal 077 from Guizhou Science and Technology Department, [2018]5764 and [2017]5724 from Guizhou Provincial Science and Technology Platform and Talent Team Project of China, and 82060228 from National Natural Science Foundation of China to Dr. X. Hu; and grant Qian Ke He J Zi [2009] 2315 from Science and Technology Fund of Guizhou Province to Dr. CX. Wei.

\section{References}

[1] N. Etminan, H.-S. Chang, K. Hackenberg et al., "Worldwide incidence of aneurysmal subarachnoid hemorrhage according to region, time period, blood pressure, and smoking prevalence in the population," JAMA Neurology, vol. 76, no. 5, pp. 588-597, 2019.

[2] M. D. Aydin, A. Kanat, C. Yolas et al., "Spinal subarachnoid hemorrhage induced intractable miotic pupil. A reminder of ciliospinal sympathetic center ischemia based miosis: an experimental study," Turkish Neurosurgery, vol. 29, no. 3, pp. 434-439, 2019.

[3] N. K. de Rooij, F. H. H. Linn, J. A. van der Plas, A. Algra, and G. J. E. Rinkel, "Incidence of subarachnoid haemorrhage: a systematic review with emphasis on region, age, gender and time trends," Journal of Neurology, Neurosurgery \& Psychiatry, vol. 78, no. 12, pp. 1365-1372, 2007.

[4] D. J. Nieuwkamp, L. E. Setz, A. Algra, F. H. H. Linn, N. K. de Rooij, and G. J. E. Rinkel, "Changes in case fatality of aneurysmal subarachnoid haemorrhage over time, according to age, sex, and region: a meta-analysis," The Lancet Neurology, vol. 8, no. 7, pp. 635-642, 2009.

[5] O. E. Batcik, A. Kanat, T. U. Cankay et al., "COVID-19 infection produces subarachnoid hemorrhage; acting now to understand its cause: a short communication," Clinical Neurology and Neurosurgery, vol. 202, article 106495, 2021.

[6] K. Kumagai, A. Tomiyama, S. Takeuchi et al., "New endovascular perforation subarachnoid hemorrhage model for investigating the mechanisms of delayed brain injury," Journal of Neurosurgery, vol. 134, no. 1, pp. 84-94, 2019.

[7] Z. V. Zheng, H. Lyu, S. Y. E. Lam, P. K. Lam, W. S. Poon, and G. K. C. Wong, "The dynamics of microglial polarization reveal the resident neuroinflammatory responses after subarachnoid hemorrhage," Translational Stroke Research, vol. 11, no. 3, pp. 433-449, 2020.

[8] C. Zhang, M. Jiang, W. Wang et al., "Selective mGluR1 negative allosteric modulator reduces blood-brain barrier permeability and cerebral edema after experimental subarachnoid hemorrhage," Translational Stroke Research, vol. 11, no. 4, pp. 799-811, 2020.

[9] B. Yuan, X. M. Zhou, Z. Q. You et al., "Inhibition of AIM2 inflammasome activation alleviates GSDMD-induced pyroptosis in early brain injury after subarachnoid haemorrhage," Cell Death \& Disease, vol. 11, no. 1, p. 76, 2020.

[10] M. Fricker, A. M. Tolkovsky, V. Borutaite, M. Coleman, and G. C. Brown, "Neuronal cell death," Physiological Reviews, vol. 98, no. 2, pp. 813-880, 2018.

[11] J. P. De Rivero Vaccari, W. D. Dietrich, and R. W. Keane, "Activation and regulation of cellular inflammasomes: gaps in our knowledge for central nervous system injury," Journal of Cerebral Blood Flow and Metabolism, vol. 34, no. 3, pp. 369-375, 2014.

[12] J. Huang, W. Lu, D. M. Doycheva et al., "IRE1 $\alpha$ inhibition attenuates neuronal pyroptosis via miR-125/NLRP1 pathway in a neonatal hypoxic ischemic encephalopathy rat model," Journal of Neuroinflammation, vol. 17, no. 1, p. 152, 2020.

[13] Q. Wu, X. L. Wang, Q. Yu et al., "Inflammasome proteins in cerebrospinal fluid of patients with subarachnoid hemorrhage are biomarkers of early brain injury and functional outcome," World Neurosurgery, vol. 94, no. 1878, pp. 472479, 2016. 
[14] X. Cheng, H. Wang, X. Zhang et al., "The role of SDF$1 / C X C R 4 / C X C R 7$ in neuronal regeneration after cerebral ischemia," Frontiers in Neuroscience, vol. 11, p. 590, 2017.

[15] L. Cui, H. Qu, T. Xiao, M. Zhao, J. Jolkkonen, and C. Zhao, "Stromal cell-derived factor-1 and its receptor CXCR4 in adult neurogenesis after cerebral ischemia," Restorative Neurology and Neuroscience, vol. 31, no. 3, pp. 239-251, 2013.

[16] C. Xie, X. Gao, Y. Luo, Y. Pang, and M. Li, "Electroacupuncture modulates stromal cell-derived factor- $1 \alpha$ expression and mobilization of bone marrow endothelial progenitor cells in focal cerebral ischemia/reperfusion model rats," Brain Research, vol. 1648, no. Part A, pp. 119-126, 2016.

[17] K. Rosenkranz, S. Kumbruch, K. Lebermann et al., "The chemokine SDF-1/CXCL12 contributes to the 'homing' of umbilical cord blood cells to a hypoxic-ischemic lesion in the rat brain," Journal of Neuroscience Research, vol. 88, no. 6, pp. 1223-1233, 2009.

[18] Y. Li, J. Huang, X. He et al., "Postacute stromal cell-derived factor- $1 \alpha$ expression promotes neurovascular recovery in ischemic mice," Stroke, vol. 45, no. 6, pp. 1822-1829, 2014.

[19] R. K. Stumm, J. Rummel, V. Junker et al., "A dual role for the SDF-1/CXCR4 chemokine receptor system in adult brain: isoform-selective regulation of SDF-1 expression modulates CXCR4-dependent neuronal plasticity and cerebral leukocyte recruitment after focal ischemia," The Journal of Neuroscience, vol. 22, no. 14, pp. 5865-5878, 2002.

[20] Y. Werner, E. Mass, P. Ashok Kumar et al., "Cxcr4 distinguishes HSC-derived monocytes from microglia and reveals monocyte immune responses to experimental stroke," Nature Neuroscience, vol. 23, no. 3, pp. 351-362, 2020.

[21] U. M. Selvaraj, S. B. Ortega, R. Hu et al., "Preconditioninginduced CXCL12 upregulation minimizes leukocyte infiltration after stroke in ischemia-tolerant mice," Journal of Cerebral Blood Flow \& Metabolism, vol. 37, no. 3, pp. 801-813, 2017.

[22] K. Roosen, M. Scheld, M. Mandzhalova, T. Clarner, C. Beyer, and A. Zendedel, "CXCL12 inhibits inflammasome activation in LPS-stimulated BV2 cells," Brain Research, vol. 1763, article 147446, no. 1763, 2021.

[23] A. Zendedel, S. Johann, S. Mehrabi et al., "Activation and regulation of NLRP3 inflammasome by intrathecal application of SDF-1a in a spinal cord injury model," Molecular Neurobiology, vol. 53, no. 5, pp. 3063-3075, 2016.

[24] C. Zhao, J. Ma, Z. Wang et al., "Mfsd2a attenuates Blood-Brain barrier disruption after Sub-arachnoid hemorrhage by inhibiting caveolae-mediated transcellular transport in rats," Translational Stroke Research, vol. 11, no. 5, pp. 1012-1027, 2020.

[25] B. J. van Dijk, J. C. M. Meijers, A. T. Kloek et al., "Complement C5 contributes to brain injury after subarachnoid hemorrhage," Translational Stroke Research, vol. 11, no. 4, pp. 678-688, 2020.

[26] T. Okada, B. Enkhjargal, Z. D. Travis et al., "FGF-2 attenuates neuronal apoptosis via FGFR3/PI3k/Akt signaling pathway after subarachnoid hemorrhage," Molecular Neurobiology, vol. 56, no. 12, pp. 8203-8219, 2019.

[27] X. Hu, J. Yan, L. Huang et al., "INT-777 attenuates NLRP3ASC inflammasome-mediated neuroinflammation via TGR5/cAMP/PKA signaling pathway after subarachnoid hemorrhage in rats," Brain, Behavior, and Immunity, vol. 91, pp. 587-600, 2021.

[28] Q. Wang, Y. Xu, J. C. Chen et al., "Stromal cell-derived factor $1 \alpha$ decreases $\beta$-amyloid deposition in Alzheimer's disease mouse model," Brain Research, vol. 1459, pp. 15-26, 2012.
[29] Y. Li, M. Niu, A. Zhao et al., "CXCL12 is involved in $\alpha$-synuclein-triggered neuroinflammation of Parkinson's disease," Journal of Neuroinflammation, vol. 16, no. 1, p. 263, 2019.

[30] W. Mao, X. Yi, J. Qin, M. Tian, and G. Jin, "CXCL12 inhibits cortical neuron apoptosis by increasing the ratio of $\mathrm{Bcl}-2 / \mathrm{Bax}$ after traumatic brain injury," The International Journal of Neuroscience, vol. 124, no. 4, pp. 281-290, 2014.

[31] Y. Fang, S. Chen, C. Reis, and J. Zhang, "The role of autophagy in subarachnoid hemorrhage: an update," Current Neuropharmacology, vol. 16, no. 9, pp. 1255-1266, 2018.

[32] Z. V. Zheng and K. C. G. Wong, "Microglial activation and polarization after subarachnoid hemorrhage," Neuroimmunology and Neuroinflammation, vol. 6, no. 1, pp. 1-12, 2019.

[33] A. M. Musluman, M. D. Aydin, A. Yilmaz et al., "The effect of degenerated neuron density of petrosal ganglion on the development of blood pressure variabilities after subarachnoid hemorrhage in a rabbit model: an experimental study," Turkish Neurosurgery, vol. 21, no. 4, pp. 559-566, 2011.

[34] S. Chen, S. Mei, Y. Luo, H. Wu, J. Zhang, and J. Zhu, "Gasdermin family: a promising therapeutic target for stroke," Translational Stroke Research, vol. 9, no. 6, pp. 555-563, 2018.

[35] N. Irrera, M. Russo, G. Pallio et al., "The role of NLRP3 inflammasome in the pathogenesis of traumatic brain injury," International Journal of Molecular Sciences, vol. 21, no. 17, p. 6204, 2020.

[36] H. Che, H. Li, Y. Li et al., "Melatonin exerts neuroprotective effects by inhibiting neuronal pyroptosis and autophagy in STZ-induced diabetic mice," The FASEB Journal, vol. 34, no. 10, pp. 14042-14054, 2020.

[37] C. Jia, H. Chen, J. Zhang et al., "Role of pyroptosis in cardiovascular diseases," International immunopharmacology, vol. 67, pp. 311-318, 2019.

[38] B. A. McKenzie, V. M. Dixit, C. Power et al., "Fiery cell death: pyroptosis in the central nervous system," Trends in Neurosciences, vol. 43, no. 1, pp. 55-73, 2020.

[39] S. Chen, Y. Zuo, L. Huang et al., "The MC4receptor agonist RO27-3225 inhibits NLRP1-dependent neuronal pyroptosis via the ASK1/JNK/p38 MAPK pathway in a mouse model of intracerebral haemorrhage," British Journal of Pharmacology, vol. 176, no. 9, pp. 1341-1356, 2019.

[40] L. Poh, S. W. Kang, S. H. Baik et al., "Evidence that NLRC4 inflammasome mediates apoptotic and pyroptotic microglial death following ischemic stroke," Brain, Behavior, and Immunity, vol. 75, pp. 34-47, 2019.

[41] Y. Dong, C. Fan, W. Hu et al., "Melatonin attenuated early brain injury induced by subarachnoid hemorrhage via regulating NLRP3 inflammasome and apoptosis signaling," Journal of Pineal Research, vol. 60, no. 3, pp. 253-262, 2016.

[42] J. Chen, C. Zhang, T. Yan et al., "Atorvastatin ameliorates early brain injury after subarachnoid hemorrhage via inhibition of pyroptosis and neuroinflammation," Journal of Cellular Physiology, vol. 236, no. 10, pp. 6920-6931, 2021.

[43] P. Xu, Y. Hong, Y. Xie et al., “TREM-1 exacerbates neuroinflammatory injury via NLRP3 inflammasome-mediated pyroptosis in experimental subarachnoid hemorrhage," Translational Stroke Research, vol. 12, no. 4, pp. 643-659, 2021.

[44] S. Wang, A. Mobasheri, Y. Zhang, Y. Wang, T. Dai, and Z. Zhang, "Exogenous stromal cell-derived factor-1 (SDF-1) suppresses the NLRP3 inflammasome and inhibits pyroptosis in synoviocytes from osteoarthritic joints via activation of the 
AMPK signaling pathway," Inflammopharmacology, vol. 29, no. 3, pp. 695-704, 2021.

[45] Z.-Y. Song, F. Wang, S.-X. Cui, and X. J. Qu, "Knockdown of CXCR4 inhibits CXCL12-induced angiogenesis in HUVECs through downregulation of the MAPK/ERK and PI3K/AKT and the Wnt/ $\beta$-catenin pathways," Cancer Investigation, vol. 36, no. 1, pp. 10-18, 2018.

[46] C. Jiang, S. Ma, R. Hu et al., "Effect of CXCR4 on apoptosis in osteosarcoma cells via the PI3K/Akt/NF- $\kappa \beta$ signaling pathway," Cellular Physiology and Biochemistry, vol. 46, no. 6, pp. 2250-2260, 2018.

[47] S. K. Daniel, Y. D. Seo, and V. G. Pillarisetty, "The CXCL12CXCR4/CXCR7 axis as a mechanism of immune resistance in gastrointestinal malignancies," Seminars in Cancer Biology, vol. 65 , pp. 176-188, 2020.

[48] https://www.researchsquare.com/article/rs-126753/v1. 